\title{
Entwässerung von teilweise zementhältigen Suspensionen - von der Rohgutcharakterisierung bis zur Anlagenumsetzung auf der Baustelle
}

\author{
Tobias Schachinger ${ }^{1}$, Andreas Böhm², Peter Reim ${ }^{3}$, Reinhold Palla ${ }^{4}$, Josef Koinig ${ }^{1}$ und Thomas Herzeg ${ }^{5}$ \\ ${ }^{1} \mathrm{SAE}, \mathrm{FB}$ Bautechnik, Tunnelbau, ÖBB-Infrastruktur AG, Wien, Österreich \\ ${ }^{2}$ Lehrstuhl für Aufbereitung und Veredlung, Montanuniversität Leoben, Leoben, Österreich \\ 3/L - Ingenieurbüro Laabmayr \& Partner ZT GesmbH, Salzburg, Österreich \\ ${ }^{4} \mathrm{Hbpm}$ Ingenieure $\mathrm{GmbH}$, Brixen, Italien \\ ${ }^{5}$ GB Projekte Neu-/Ausbau, Projektleitung Koralmbahn, ÖBB-Infrastruktur AG, Klagenfurt, Österreich
}

Eingegangen 2. Juli 2019; angenommen 12. Juli 2019; online publiziert 30. Juli 2019

Zusammenfassung: Im Baulos 60.3 - St. Kanzian der Koralmbahn liegen die beiden Tunnelbauwerke Untersammelsdorf und Srejach in glazialen Stillwassersedimenten („Seeton“). Für die Errichtung waren zur Vergütung des anstehenden Bodens umfangreiche Spezialtiefbaumaßnahmen als Kombination von Bohrpfählen und Düsenstrahlverfahren (DSV) erforderlich.

In den Jahren 2008-2009 wurde durch die ÖBB im Nahbereich der späteren Tunnelbauwerke als eigene Baumaßnahme das Versuchsfeld Untersammelsdorf errichtet.

Damit sollten für die Bauausschreibung und spätere Herstellung der Tunnelbauwerke Erkenntnisse zu erreichbaren Eigenschaften (v.a. Durchmesser und Druckfestigkeit) des DSV-Körpers in den Stillwassersedimenten sowie zur Menge, Beschaffenheit und Entwässerbarkeit des systembedingten Rücklaufs (einer Suspension aus Wasser, gelöstem Boden und Bindemittel in wechselnden Anteilen) gewonnen werden.

Ein Ergebnis war, dass der Rücklauf nur durch Schwerkraft alleine - wie sonst bei zahlreichen Spezialtiefbaumaßnahmen üblich - praktisch nicht entwässerbar ist.

Aus diesem Grund wurden zusammen mit dem Lehrstuhl für Aufbereitung und Veredlung der Montanuniversität Leoben in einem systematischen Untersuchungsprogramm vom Labor bis zum Pilotversuch die für die Auswahl von geeigneten Entwässerungsapparaten notwendigen Parameter der Suspension (Feststoffanteil, Viskosität, Filtrationswiderstand) und des Feststoffes in seinem Körnungs- und mineralogischen Aufbau erarbeitet. Nicht nur

\footnotetext{
Ass. Prof. Dipl.-Ing. Dr. mont. A. Böhm ( $\varangle)$ Lehrstuhl für Aufbereitung und Veredlung, Montanuniversität Leoben,

Franz-Josef-Straße 18,

8700 Leoben, Österreich

andreas.boehm@unileoben.ac.at
}

die Schwankung im Feststoffanteil und Körnungsanteil, sondern auch die bauvorhabenbedingte Vermischung mit Zement war zu berücksichtigen. Der Einfluss des Zementes, während der Entwässerung und im Sediment von Schwer- und Fliehkraftentwässerung und im Filterkuchen wird erläutert. Der Vorschlag zum stufenweisen Aufbau des Entwässerungssystems, wie er Eingang in die Rohbauausschreibung gefunden hat, wird der Bauausführung durch die ausführenden Firmen gegenübergestellt.

Schlüsselwörter: Untersammelsdorf, Srejach, Seeton, Düsenstrahlverfahren, Rücklauf, Entwässerung, Kammerfilterpresse, Dekanter

Dewatering of Partly Cement Containing Suspensions-from Materials Characterisation to Plant Realization

Abstract: In construction lot 60.3-St. Kanzian of the Koralmbahn, the two railway tunnels Untersammelsdorf and Srejach are located in glacial lacrustine clay sediments ("Seeton"). To cope with these soils during tunnel works, a combination of bored piles and jet grouting was necessary to a large extent. During the years 2008-2009, a test construction site ("Versuchsfeld Untersammelsdorf") was built by ÖBB. This separate construction lot was situated close to the later Untersammelsdorf tunnel. ÖBB's intention was to gain information on tendering and future construction of the railway tunnels, especially regarding the properties of the jet grouting columns (mainly reachable diameter and compressive strength) in "Seeton". A second aim was to get information on the backflow (a mixture of eroded soil, water and cement), e.g. its amount and properties, particularly regarding dewatering. 
One early conclusion was that gravity-as it is common practice in many jet grouting activities - is not sufficient for sedimentation of the fine grained clay minerals.

To overcome the problem, a systematic investigation programme from lab to pilot scale was launched in cooperation with the Chair of Mineral Processing at Montanuniversitaet Leoben to elaborate the parameters of suspension (solid concentration, viscosity, sedimentation flux, resistance to filtration) and the solids concerning size distribution and mineralogical composition. Not only the variability had to be taken into account, but also the influence of admixed cement. The influence of cement during dewatering and in the solidification of the sediment dewatered by gravity and in the centrifugal field as well as in the filter cake is explained. The proposal of a system for stepwise mechanical dewatering is presented, which was part of the tendering documents. During construction works, dewatering of the backflow was made according to the client's proposal,which is also presented in this paper.

Keywords: Untersammelsdorf, Srejach, Lacustrine clay, Jet grouting, Backflow, Dewatering, Filter press, Decanter

\section{Einleitung}

Die Koralmbahn stellt eine neue zweigleisige Hochleistungsstrecke zwischen Graz und Klagenfurt dar. Sie besitzt eine Länge von ca. $130 \mathrm{~km}$. Herzstück ist der $33 \mathrm{~km}$ lange Koralmtunnel; darüber hinaus werden zwölf neue Bahnhöfe und Haltestellen, über 100 Brücken und Unterführungen sowie zahlreiche Tunnelbauwerke errichtet.

Im Kärntner Abschnitt Mittlern-Althofen/Drau werden sechs Tunnelbauwerke zur Tunnelkette St. Kanzian zusammengefasst. Zwei Tunnelbauwerke dieser Tunnelkette (Tunnel Srejach und Tunnel Untersammelsdorf) liegen teilweise in glazialen Stillwassersedimenten ("Seeton“) und erforderten daher umfangreiche Spezialtiefbaumaßnahmen [1-4], die auch aufbereitungstechnische Fragestellungen in Form der Entwässerung von teilweise zementhaltigen Suspensionen beinhalteten. Im gegenständlichen Beitrag wird darüber berichtet.

\section{Geologie, durchgeführte Baumaßnahmen und Problemstellung}

Die Tunnelkette St. Kanzian befindet sich in den östlichen Ausläufern des Klagenfurter Beckens. Dieses wird aufgebaut von verschiedenen paläozoischen und triassischen Festgesteinen, auf die im Miozän feinklastische, teilweise kohleführende Sedimente und Konglomerate abgelagert wurden. Im Quartär erfolgte die geomorphologische Prägung der Landschaft. Die quartären Ablagerungen bilden durch den wiederholten Vorstoß und Rückzug des Draugletschers einen Wechsel von eiszeitlichen Moränensedimenten und fluvioglazialen Ablagerungen. In den Toteislöchern kam es zur Ablagerung mächtiger Stillwassersedimente („Seeton“). Nach dem endgültigen Rückzug der Gletscher

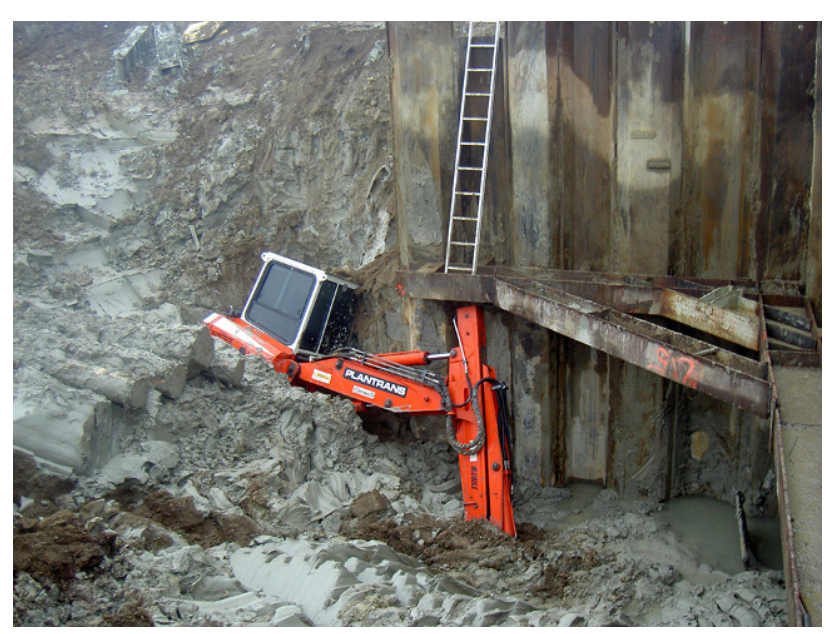

Abb. 1: Versagende Baugrube im Seeton im Bereich der Koralmbahn

wurden von den Flüssen mächtige Schotterterrassen und Sanderflächen geschüttet.

Bei den Seetonen gibt es Sand- und Schluff-Ton-dominierte Zonen. Ihre Konsistenz variiert von breiig, weich bis steif, wodurch es sich um einen in geotechnischer Hinsicht äußerst ungünstigen Baugrund handelt (Abb. 1).

Es wurden Mächtigkeiten dieser Ablagerungen bis über $40 \mathrm{~m}$ erkundet.

Der Tunnel Srejach ist eines der beiden im Seeton liegenden Tunnelbauwerke. Er weist eine Länge von $620 \mathrm{~m}$ auf. Da keine Unterkante des Seetons erkundet wurde, ist er schwimmend gegründet. Er wurde in Deckelbauweise mit einer aufgelösten Bohrpfahlwand beidseits des späteren Tunnels hergestellt. Zur Aussteifung der beiden Bohrpfahlwände wurde unterhalb der späteren Sohlplatte eine 2-3m dicke Bodenvergütung mittels des Düsenstrahlverfahrens (DSV) vorab von über Tage hergestellt.

Das Düsenstrahlverfahren (DSV) wird gemäß ÖNORM EN 12716 [5] als Vorgang bezeichnet:

der darin besteht, dass Boden oder mäßig festes Gestein in seine Bestandteile zerlegt und mit zementhaltiger Mischung versetzt bzw. teilweise durch diese Mischung ersetzt wird. Das Auflösen des Bodengefüges wird durch einen energiereichen Flüssigkeitsstrahl bewirkt, wobei die Flüssigkeit die Zementsuspension sein kann.

Mit diesem Verfahren können zylindrische Elemente, sogenannte DSV-Säulen, hergestellt werden. Durch das Aneinanderreihen mehrerer Säulen entstehen DSV-Körper.

Das zweite Tunnelbauwerk, der Tunnel Untersammelsdorf, schließt bei einer Länge von $665 \mathrm{~m}$ neben Stillwassersedimenten in der Sohle auch quartäre Kiese und Sande und Moränensedimente auf. Aufgrund einer maximalen Überlagerung von $33 \mathrm{~m}$ über Schienenoberkante und der ungünstigen geotechnischen Bedingungen wurde der Großteil des Tunnels in geschlossener Bauweise mit einem Gewölbequerschnitt wie folgt hergestellt: Zur Ausbildung eines "Schutzgewölbes in der Firste" und um ein Ausfließen von sandigen Lagen aus der Firste im Ausbruchbereich zu vermeiden, erfolgte die Herstellung eines bis $3 \mathrm{~m}$ dicken DSV-Firstgewölbes von über Tage. Zur Verspannung 
Abb. 2: Luftbild des Versuchsfeldes Untersammelsdorf während des Aushubs zwischen den Bohrpfählen

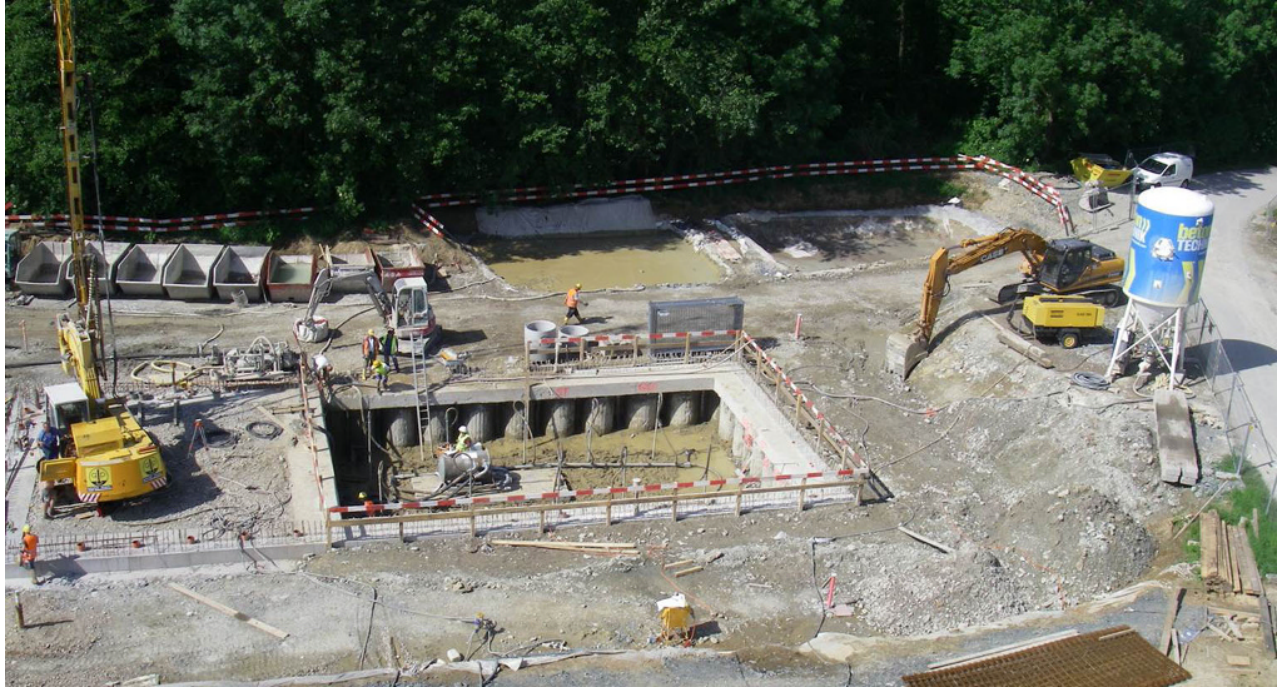

und Auflagerung dieses DSV-Firstgewölbes und zur Vermeidung des Ausfließens im Ulmenbereich wurden beidseitig Bohrpfähle hergestellt, die in den tragfähigen Untergrund einbinden. Im Schutz dieser Spezialtiefbaumaßnahmen erfolgte dann der eigentliche Vortrieb.

\section{Versuchsfeld Untersammelsdorf}

Bereits aus den Erfahrungen früherer Bauvorhaben in der Region bzw. aus den Laborwerten der Bodenproben war dem Bauherrn die geotechnische Problematik der dargelegten geplanten Baumaßnahmen bewusst. Bei der Planung resultierten daraus sehr hohe Bauwerkskosten, die v. a. auf das DSV-Verfahren als den Kostentreiber zurückzuführen waren. Insgesamt war geplant, bei beiden Tunnels in Summe rd. $87.000 \mathrm{~m}^{3}$ DSV-Körper herzustellen.

In den Jahren 2008-2009 wurde daher durch die ÖBB im Nahbereich der späteren Tunnelbauwerke als eigene Baumaßnahme das Versuchsfeld Untersammelsdorf errichtet (Abb. 2; [6]).

Damit sollten für die Bauausschreibung und spätere Herstellung der Tunnelbauwerke Erkenntnisse zu erreichbaren Eigenschaften (v.a. Durchmesser und Druckfestigkeit) des DSV-Körpers in den Stillwassersedimenten sowie zur Menge, Beschaffenheit und Entwässerbarkeit des systembedingten Rücklaufs (einer Suspension bzw. Trübe aus Wasser, gelöstem Boden und Bindemittel in wechselnden Anteilen, die im Ringraum zwischen Bohrlochrand und Bohr-/Düsgestänge wieder ausgetragen wird) gewonnen werden. Im Schutze eines $25 \mathrm{~m}$ mal $10 \mathrm{~m}$ großen Bohrpfahlkastens wurden dabei zahlreiche DSV-Säulen und -Körper hergestellt und später ausgegraben sowie ein kurzer Tunnelvortrieb durchgeführt.

Hinsichtlich des DSV-Rücklaufs konnte ein Rücklauffaktor von ca. 3,5 ermittelt werden. Dies bedeutet, dass die Rücklaufmenge mindestens das 3,5-fache der herzustellenden DSV-Kubatur beträgt. Außerdem wurde festgestellt, dass der Rücklauf nur durch Schwerkraft alleine - wie sonst bei zahlreichen Spezialtiefbaumaßnahmen üblich - prak- tisch nicht sedimentiert. Erschwerend für die Planung der für die Deponierung des Feststoffinhaltes notwendigen Entwässerungsanlage kommt die Wirkung des teilweise in der Rücklauftrübe enthaltenen Zements hinzu.

Um eine entsprechende Planungssicherheit für die Ausschreibung und Ausführung zu gewinnen, wurden zusammen mit dem Lehrstuhl für Aufbereitung und Veredlung der Montanuniversität Leoben umfangreiche Untersuchungen zur Entwässerung des DSV-Rücklaufs durchgeführt.

\section{Probenahmetätigkeiten}

Aufgrund der baugeologischen Randbedingungen (Böden unterschiedlicher Eigenschaften entlang des Verlaufes der beiden Tunnels) war eine wesentliche Randbedingung für die Entwässerungsanlage, dass eine möglichst gute Anpassung an die auftretenden Suspensions- und Körnungsbedingungen und ihr betriebsbedingtes Schwankungsverhalten möglich ist. Ein Apparat alleine kann nicht alle Anforderungen in technischer wie auch wirtschaftlicher Sicht erfüllen. Dementsprechend war für den störfallfreien, kostengünstigen Betrieb eine Abfolge von geeigneten Entwässerungsapparaten zur stufenweisen Reduktion des Wassergehaltes bis zur deponiefähigen Restfeuchte zu planen.

Der Beschaffung planungsrelevanter Proben kam dabei große Bedeutung zu.

In systematischer Herangehensweise wurden noch während des Betriebes des Versuchsfeldes erste Proben direkt am Bohrlochmund für die DSV-Säulen und vom Zulauf zu den Mulden für den DSV-Rücklauf durch Mitarbeiter des Lehrstuhls gezogen, um sowohl die Suspension als auch den Feststoff hinsichtlich der Entwässerungseigenschaften zu untersuchen. Die im Versuchsfeld zugänglichen feinstkörnigen, tonig-schluffigen Stillwassersedimente stellten die energieintensivste Entwässerungsaufgabe dar und ließen eine Apparatevorauswahl zu.

In einem zweiten Schritt wurden mit Hilfe vorhandener Kernbohrungen charakteristische Proben aus zukünftigen Tunnelabschnitten ausgewählt, um die Variabilität in Art 


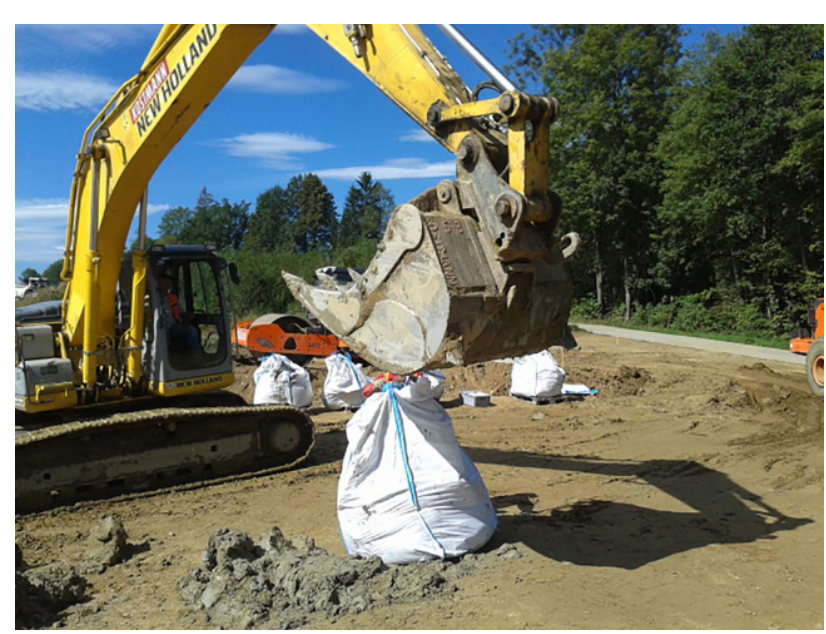

Abb. 3: Gewinnung von Probematerial für Pilotversuche

und Menge der Bodenarten (kiesig-sandig bis tonig-schluffig), aber auch die Wirkung des Zementanteils in das Sedimentations- und Filtrationsverhalten miteinzubeziehen.

In einem dritten Schritt wurden in einem eigenen Schurfprogramm 6t Probenmaterial für Pilotversuche bei Herstellern von als zweckmäßig beurteilten Apparaten derart erarbeitet, dass ausreichend Aufgabegut mit vergleichbaren entwässerungstechnischen Eigenschaften verfügbar war (Abb. 3).

\section{Kleinversuche zur Charakterisierung von Suspension und Feststoff}

An den ersten beiden Probentypen wurden die für die Auswahl und Auslegung von Entwässerungsapparaten maßgeblichen Suspensions- und Feststoffparameter unter besonderer Berücksichtigung der Zementwirkung erarbeitet.

\subsection{Untersuchungsmethoden und Kennwerte}

Die Feststoffmengenanteile in der (Rücklauf-)Trübe ergaben sich aus Trocknungsversuchen bis zur Gewichtskonstanz. Die Trübeviskosität wurde bei der höchsten Feststoffvolumenkonzentration mit einem Rotationsviskosimeter (Typ Haake Viskotester 550) gemessen, für die Veränderung der Viskosität mit der Feststoffvolumenkonzentration kam das Kapillarviskosimeter nach Steiner zum Einsatz. Einstabmessketten bzw. Glaselektroden dienten zur Bestimmung des pH-Wertes und der Leitfähigkeit der Trübe. Das Klärverhalten wurde in Absetzreihenversuchen mit der Betriebstrübe ermittelt, während der Filtrationswiderstand aus Laborversuchen mit entsprechenden Apparaturen für Saug- und Druckfiltration am Lehrstuhl für Aufbereitung und Veredlung erarbeitet wurde.

$$
\operatorname{gradp}=r \cdot \eta \cdot \frac{\dot{V}}{A}
$$

$\operatorname{grad} p=$ Gradient des Drucks über den Filterkuchen $[\mathrm{Pa} / \mathrm{m}]$, spezifischer Filtrationswiderstand $\left[1 / \mathrm{m}^{2}\right]$, dynamische Viskosität [Pa.s],

$\dot{V}=\quad$ Volumenstrom Filtrat $\left[\mathrm{m}^{3} / \mathrm{s}\right]$,

$A=\quad$ Filterfläche $\left[\mathrm{m}^{2}\right]$.

Die Definition für den Filtrationswiderstand ist in GI. 1 („Darcy-Gleichung“) angegeben. Da vorab nicht die Auslegung des Filterapparates im Vordergrund stand, sondern die Ermittlung einer Kennzahl zum Vergleich der einzelnen Probentypen hinsichtlich ihrer Filtrierbarkeit mit und ohne Einfluss von Zement, wurde der Widerstand am ausgebildeten Kuchen bei zeitlich konstanten Druckverhältnissen gemessen.

Die Korngrößenverteilungen entstammen einer Kombination aus Nass-Siebanalyse kombiniert mit Sedimentationsanalyse am Körnungsanteil unter $25 \mu \mathrm{m}$. Die Kornform wurde aus der mineralogischen Untersuchung und Beobachtungen im Stereomikroskop abgeschätzt.

Die Feststoffdichte wurde mit dem Helium-Gaspyknometer ermittelt und diente vor allem zur Umrechnung des direkt bestimmten Feststoffgehaltes in häufig gebrauchte Feststoffmengenangaben.

Die Feststoffmengenangaben werden in der Terminologie und der Definition nicht in allen Ingenieurwissenschaften gleich verwendet. Deshalb war es bei fachübergreifender Zusammenarbeit notwendig, die Definitionen vorab klar anzugeben. In der Aufbereitungstechnik beispielsweise werden Konzentrationsangaben immer als Volumenbezug verstanden, während Gehalte massebezogene dimensionslose Größen ( $\mathrm{m} \%$ ) sind. Ein Feststoffgehalt wird in der Aufbereitungstechnik bezogen auf die Gesamttrübemasse ermittelt. In der Geotechnik wird hingegen die Trockenmasse als Bezugsmasse herangezogen, was in der aufbereitungstechnischen Terminologie einer Beladung entspricht. Vor allem in der Kommunikation mit Herstellern von Entwässerungsapparaten, die die Feststoffmengenangaben zwingend als auslegungsrelevante Daten benötigen, war daher immer höchste Transparenz bei den Begriffen geboten.

\subsection{Ergebnisse der Laborversuche $[7,8]$ und Maschinenvorauswahl}

\subsubsection{Feststoffanteil und seine Schwankung}

Die Feststoffmassekonzentration (Masse Feststoff bezogen auf das Volumen Trübe) der direkt am Bohrloch gezogenen Proben des DSV-Rücklaufs liegt im arithmetischen Mittel aus 7 Proben bei $656 \pm 152 \mathrm{~kg} / \mathrm{m}^{3}$ (Feststoffgehalt: $45,8 \pm 7,03 \%)$, jene der in die Absetzmulden verpumpten Proben bei $442 \pm 190 \mathrm{~kg} / \mathrm{m}^{3}$ (Feststoffgehalt: $33,5 \pm 9,97 \%$ ). Der niedrigste Wert der verpumpten Proben liegt bei $310 \mathrm{~kg} / \mathrm{m}^{3}$, der höchste bei $924 \mathrm{~kg} / \mathrm{m}^{3}$. Sobald die Proben mit Zement in Kontakt kommen, steigt der pH-Wert der Trübe von 8 auf Werte zwischen 11,5-12, womit auch die Leitfähigkeit von etwa 300 auf $1200 \mu \mathrm{S} / \mathrm{cm}$ ansteigt. 
Die Trübe zeigt strukturviskoses Verhalten. Bei einer Massekonzentration von $644 \mathrm{~kg} / \mathrm{m}^{3}$ sinkt die Viskosität von $7 \mathrm{~Pa} \cdot \mathrm{s}$ bei einem Schergefälle von $10 \mathrm{~s}^{-1}$ auf etwa $0,25 \mathrm{~Pa} \cdot \mathrm{s}$ bei $600 \mathrm{~s}^{-1}$. Bei etwa der halben Feststoffkonzentration sinkt die scheinbare Viskosität auf $7 \mathrm{mPa} \cdot \mathrm{s}$ (gemessen mit dem Kapillarviskosimeter).

\subsubsection{Mineralogische Zusammensetzung und Körnungsaufbau}

Der hohe Anteil an Schichtsilikaten - Muskowit (36\%), Chlorit $(11 \%)$, Smektit (2\%) und Kaolinit (2\%) - im Seeton ist einerseits für das Fließverhalten verantwortlich, andererseits bedingt die Plättchenform neben der Kornverteilung den hohen Filtrationswiderstand. Der verbleibende Mineralbestand setzt sich aus Quarz (18\%), Dolomit (12\%), Plagioklas (9\%) und etwas Pyrit (1\%) zusammen [9].

Den Körnungsaufbau der Feststoffinhalte aus den unterschiedlichen Bodenbereichen "kiesig“ (Probe ÖBB 3), "sandig“ (Probe ÖBB 1), "tonig-schluffig“ (Probe ÖBB 2 und 4) und seine Schwankung zeigen Abb. 4 und Tab. 1. Der Vorerkundung entsprechend macht der tonig-schluffige Bereich etwa $2 / 3$ der Sedimente im Bereich der Bohrpfähle des benachbarten Bauloses 60.2 aus. $99 \%$ sind kleiner als $63 \mu \mathrm{m}$. Der Anteil kleiner $2 \mu \mathrm{m}$ liegt zwischen 20 und $25 \%$.

Die Korngrößenverteilung des in der Versuchstätigkeit durchgehend verwendeten Zementes (CEM II B-M (SL), Stoffdichte $2,92 \mathrm{~g} / \mathrm{cm}^{3}, 4400 \mathrm{~cm}^{2} / \mathrm{g}, 28$ Tage Festigkeit: $44 \mathrm{~N} / \mathrm{mm}^{2}$, Fa. Rohrdorfer) entspricht nahezu jener der tonig-schluffigen Probe 2. In Abb. 4 sind zusätzlich jene von Dorr Oliver [10] angegebenen Grenzen zwischen Saugfiltration der mit Unterdrücken um 1 bar und Druckfiltration der mit Überdruck > 1 bar arbeitenden Apparate angegeben.

Der parallele Verlauf des Feinkornanteils $<25 \mu \mathrm{m}$ der aus Bohrkernen generierten Proben (ÖBB 1-4) mit jenen der direkt im Betrieb des Versuchsfeldes gezogenen zeigt einerseits, dass die Probenverarbeitung der ausgetrockneten Bohrkernproben vertrauenswürdig ist, und andererseits die geologische Beziehung zwischen den Bodentypen.

Die mittlere Feststoffdichte der Trockensubstanz, bestimmt mit dem Heliumgaspyknometer, beträgt $2800 \mathrm{~kg} / \mathrm{m}^{3}$.

Der Körnungsaufbau wie auch die Feststoffvolumenkonzentration schwanken stark. Die Konzeption einer Entwässerungsanlage bzw. eines Entwässerungsaggregates muss also geeigneten Maschinenschutz durch Grobkornabscheidung oder geeignet robuste (verschleißfeste) Maschinenkomponenten aufweisen. Der hohe Anteil an plattigen Schichtsilikaten sowie auch die Durchgangsvertei-

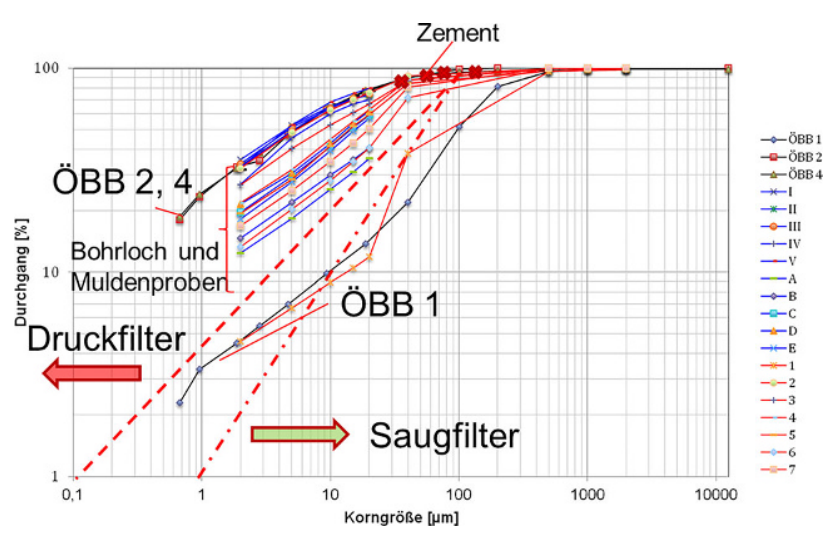

Abb. 4: Darstellung der Körnungsverteilungen der verarbeiteten Proben sowie der korngrößenverteilungsbedingten Zuordnung von Entwässerungstechnik $[7,8,10]$

lung deuten bereits Druckfiltration als einzige zielführende Entwässerungsform an.

\subsubsection{Sedimentationsverhalten bei Schwerkraft- entwässerung}

Die Klärversuche wurden ohne Trübeteilung direkt mit den Eingangstrüben im Entnahmegebinde (10I Kübel) durchgeführt. Das Klärverhalten der zementfreien Suspensionen beschrieben durch die Klärgeschwindigkeit und die Klärleistung (GI. 2) schwankt in Abhängigkeit von der Feststoffmassekonzentration und dem Körnungsaufbau von einer Klärgeschwindigkeit von $1,62 \mathrm{~mm} / \mathrm{h}$ bei $970 \mathrm{~kg} / \mathrm{m}^{3}$ $\left(157 \mathrm{~kg} / \mathrm{m}^{2} \cdot \mathrm{h}, 38,2 \%<40 \mu \mathrm{m}, 4,6 \%<2 \mu \mathrm{m}\right)$ bis $0,18 \mathrm{~mm} / \mathrm{h}$ bei $644 \mathrm{~kg} / \mathrm{m}^{3}\left(12 \mathrm{~kg} / \mathrm{m}^{2} \cdot \mathrm{h}, 90 \%<40 \mu \mathrm{m}, 29 \%<2 \mu \mathrm{m}\right)$. Die Zugabe von anionischen Flockungsmitteln bis zu einer Konzentration von $90 \mathrm{~g} / \mathrm{t}$ verbessert das Klärverhalten bei etwa halber Feststoffkonzentration der tonig-schluffigen Proben von $340 \mathrm{~kg} / \mathrm{m}^{3}$ von 3,36 auf $11,1 \mathrm{~mm} / \mathrm{h}$. Dies entspricht einem Rundeindicker von etwa $2500 \mathrm{~m}^{2}$ Fläche bzw. $60 \mathrm{~m}$ Durchmesser. Zement führt zu einer Erhöhung des $\mathrm{pH}$-Werts auf etwa 12 und zur Flockung der Feinanteile.

$$
\Psi=c \stackrel{\circ}{ } V_{K}
$$

$\Psi=$

$c=$

$v_{k}=$ flächenbezogener Feststoffmassestrom $\left[\mathrm{kg} /\left(\mathrm{m}^{2} \cdot \mathrm{h}\right)\right]$

Feststoffmassekonzentration $\left[\mathrm{kg} / \mathrm{m}^{3}\right]$, Klärgeschwindigkeit [m/s].

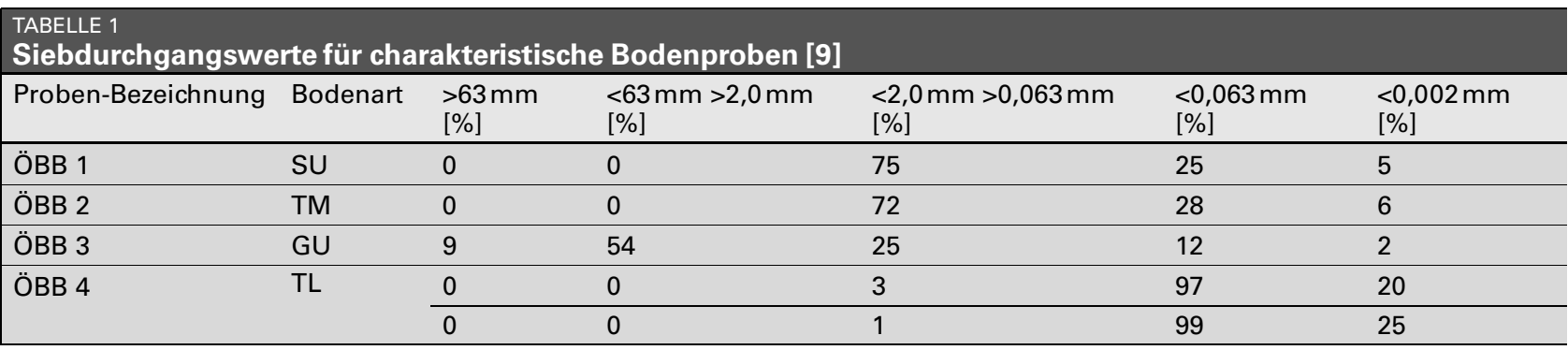


Die Feststoffmassekonzentration des Rücklaufs am Bohrlochmund entspricht für das tonig-schluffige Material weitestgehend bereits dem möglichen Eindickungsgrad eines Schwerkrafteindickers. Das Sediment dieses Bodentyps bleibt auch nach mehrwöchigem Schwerkrafteinfluss pumpbar. Die Feststoffkonzentration liegt in einem für Kammerfilterpressen günstigen Bereich. Ein mechanischer Eindicker zur Vorentwässerung bzw. Klarwasserrückgewinnung hat keinen positiven Effekt.

Die Ausnutzung der Stabilität des DSV-Rücklaufs zur Speicherung mit oder ohne Rührwerk in einem einfachen Becken bzw. Bodenaushub verbleibt als wesentlicher Aspekt für die Planung des Entwässerungssystems. Dadurch lässt sich der Betrieb des Düsenstrahlverfahrens von der Entwässerung entkoppeln.

Auch bei einer baustellenbedingten Verdünnung der am Bohrlochmund anfallenden Suspension auf etwa $350 \mathrm{~kg} / \mathrm{m}^{3}$ bleibt nach Verpumpen die Stabilität (bei pH 12) für eine Verweilzeit von mindestens $8 \mathrm{~h}$ in einem Becken ohne extern eingebrachte Scherkräfte (beispielsweise durch Umpumpen oder ein Rührwerk) erhalten.

Die Frage der Verfestigung eines Sedimentes bei hohem Zementanteil wurde in einer eigenen umfangreichen Untersuchungsreihe für die einzelnen Bodentypen behandelt [8] In systematischen Absetz-Reihenversuchen in Standzylindern mit 1 I Fassungsvermögen mit einer Dauer von 1, 2, 3 und 7 Tagen wurde jeweils die Feststoffkonzentration zwischen 150 und $750 \mathrm{~kg} / \mathrm{m}^{3}$ in Schritten von $150 \mathrm{~kg} / \mathrm{m}^{3}$ gestaffelt und die Trockensubstanz wiederum in $25 \mathrm{~m} \%$ Stufen durch Zement ersetzt. Die Sedimente jedes einzelnen Versuches wurden durch einfachste Bemusterung auf ihr Festigkeitsverhalten hin untersucht. Erst bei Zementgehalten über $50 \%$ und Feststoffgehalten von $600 \mathrm{~kg} / \mathrm{m}^{3}$ für sandige Proben stellte sich eine sichtbare, wenn auch nicht dauerhafte Verfestigung ein. Durch Kneten des Sediments in der Hand (Scherbeanspruchung) verflüssigte sich der Kuchen wieder. Für tonig-schluffige Proben konnte der Effekt ab $75 \mathrm{~m} \%$ Zement und einer Konzentration von $750 \mathrm{~kg} / \mathrm{m}^{3}$ beobachtet werden. Der Grund für die Fließbewegung auf Scherbeanspruchung liegt im überhöhten Verhältnis zwischen Wasser zu Zement. Zum Vergleich sei angeführt, dass bei der Betonherstellung ein Wasser zu Zement- bzw. Bindemittel-Verhältnis von etwa 0,6 gewählt wird. Im DSVRücklauf ergaben sich w/z-Werte zwischen 1,5 und 2, womit keine bleibende Verfestigung möglich ist.

Der Entwässerung kommt also neben der Fest-/FlüssigTrennung auch die Aufgabe zu, das Verhältnis zwischen Wasser und Zement im Filterkuchen auf einen Wert zu bringen, der eine verfestigende Wirkung des abgebundenen Zements zulässt. Dies legt die zulässige Restfeuchte des Kuchens fest.

\subsubsection{Filtrationsversuche}

Versuche zur Scher- und Druckfestigkeit des Filterkuchens wurden in Zusammenarbeit mit dem Baustoffinstitut Camillo Sitte in Wien durchgeführt [9]. Mit systematischen Laborfiltrationsversuchen bei 7 bar Pressdruck, vorgegebenem Trübevolumen von $370 \mathrm{~cm}^{3}$ und $450 \mathrm{~kg} / \mathrm{m}^{3}$ Feststoff- massekonzentration wurde vorab das Verhalten der Suspension bei unterschiedlichen Zementanteilen untersucht.

Ohne Zementzugabe weist die Probe (Filtrationswiderstand von $6,03 \cdot 10^{15} \pm 1,65 \cdot 10^{14} 1 / \mathrm{m}^{2}$ ) bei einer Filtrationsdauer von 220 min eine minimale Kuchenrestfeuchte von $21 \mathrm{~m} \%$ auf. Bei sofortiger Verarbeitung der Trübe bewirkt Zement bei $25 \mathrm{~m} \%$ Zementanteil eine drastische Verringerung der Filtrationszeit auf $23 \mathrm{~min}$ und eine minimale Restfeuchte von $27 \mathrm{~m} \%$. Bei einem erlaubten Restwassergehalt von $33 \mathrm{~m} \%$ sinkt die Filtrationszeit unter sonst gleichen Bedingungen auf $13 \mathrm{~min}$. Erhöht sich die Verarbeitungszeit (Stehzeit) der Trübe vor dem Filtrieren um $2 \mathrm{~h}$, halbiert sich die Filtrationszeit noch einmal. Diesem Verhalten sollte in den Pilotversuchen noch spezielle Aufmerksamkeit geschenkt werden.

Bei einem Restwassergehalt von etwa $32 \%$ weist der Kuchen eine Druckfestigkeit nach 7 Tagen von 2,6 MPa auf. Damit wurde die Restfeuchte auf einen Wert von $32,5 \pm 2,5 \%$ festgelegt. In der Aufgabe ist mit $300-600 \mathrm{~kg} / \mathrm{m}^{3}$ Feststoff mit einem maximalen Zementanteil von $25 \%$ zu rechnen. Die dominante Bodenkomponente weist hohen Feinanteil auf mit $20-25 \%<2 \mu \mathrm{m}$ und $90 \%<40 \mu \mathrm{m}$. Mit Spritzkorn bis $10 \mathrm{~mm}$ ist zu rechnen.

Aufbauend auf diesen Eckdaten war eine Vorcharakterisierung der Apparate möglich.

\subsubsection{Apparatevorauswahl}

Kammerfilterpressen, Vollmantelschneckenzentrifugen bzw. Dekanter und Siebbandpressen wurden auf der Basis der technischen Eignung und einer ersten Abschätzung der Investitions- und Betriebskosten verglichen.

In der kontinuierlich arbeitenden Siebbandpresse wird ein durch den Einsatz von Flockungsmitteln scherfest gemachter Kuchen zwischen endlos umlaufenden Siebbahnen in mehreren Stufen auf Scherung und Druck belastet. Ohne näher auf die dem Entwässerungsgrad angepassten Druckniveaus des scherstabilen Filterkuchens einzugehen, liegt der systemimmanente Verbrauch an Flockungsmitteln eine Größenordnung über dem Verbrauch in anderen Entwässerungsaggregaten.

Für die Siebbandpresse muss aufgrund der beschränkten Filterkuchenhöhe und exakt einzuhaltender Verdichtungsgeschwindigkeit je Entwässerungszustand nicht nur die Trübemenge, sondern auch die Feststofffracht für eine Maschine eingehalten werden. Aufgrund der Umschlingung von Presswalzen am Walzenstuhl ist die Verschleißfestigkeit des Siebbandes an die Einhaltung von Kornobergrenzen gebunden. Zement oder hoher pH-Wert haben keine bekannte nachteilige Wirkung.

Der in der Baubranche als Dekanter, in der Verfahrenstechnik als Vollmantelschneckenzentrifuge eingeführte Apparat ist jene Maschine zur kontinuierlichen Sedimentation im Fliehkraftfeld, die umso besser arbeitet, je geringer der Feststoffgehalt in der Aufgabe ist. Feststoffmassekonzentrationen bis zu einer Obergrenze von $350 \mathrm{~kg} / \mathrm{m}^{3} \mathrm{kön}$ nen verarbeitet werden, darüber hinaus muss durch $\mathrm{Zu}$ satzwasser (!) verdünnt werden. Der Feststoffinhalt darf einen gewissen Wert nicht übersteigen, die Trübemenge darf jedoch in vergleichsweise weiten Bereichen schwan- 
ken. Flockungsmittel unterstützen die Feststoffentfrachtung des Klarwassers, erhöhen aber die Kuchenrestfeuchte. Die hohe installierte Leistung führt zu hohen Energiekosten. Die Strategie gegen Verschleiß wird von Hersteller zu Hersteller unterschiedlich gehandhabt. Sowohl Verschleißschutz des mit bis zu $5000 \mathrm{~min}^{-1}$ umlaufenden Außenmantels und der mit geringer Differenz $\left( \pm 50 \mathrm{~min}^{-1}\right)$ drehenden Schnecke, wie auch Oberkornbegrenzung durch Vorklassierung sind möglich. Eine exakte Reinigung mit Zusatzwasser vor jedem Abschaltvorgang ist unbedingt erforderlich, um Unwucht zu vermeiden.

Kammerfilterpressen werden für unterschiedliche Druckniveaus angeboten. Günstige Maschinen arbeiten bis etwa 7 bar mit Zentrifugalpumpen, für höhere Pumpdrücke müssen die Maschinen massiver und mit anderen Pumpen ausgeführt werden. Darüber hinaus lässt sich der Entwässerungserfolg mit Druckluft beaufschlagten Gummimembranen in den Filterrahmen erhöhen. Kammerfilterpressen funktionieren umso wirkungsvoller, je höher die Ausgangsfeststoffkonzentration ist. Für Kammerfilterpressen stellt eine Feststoffmassekonzentration von $350 \mathrm{~kg} / \mathrm{m}^{3}$ bei der feinen Körnung eine massive Erhöhung der Zykluszeit und damit erhöhte Maschinenkosten (Verlust an Durchsatzleistung) dar, der man - sofern möglich - mit dem Einsatz von Flockungsmitteln begegnen kann. Die Presszeiten sollten $15 \mathrm{~min}$, die Zykluszeiten $20 \mathrm{~min}$ nicht überschreiten. Je nach Hersteller sind die Angaben über die zulässigen Oberkorngrößen im Spritzkorn unterschiedlich: 3-10 mm sollten bei einem Gehalt kleiner als $5 \mathrm{~m} \%$ in der Aufgabe zum Erhalt der Standzeit der Filtertücher nicht überschritten werden. Mit Salzsäure angesäuertes Waschwasser (5\%-ige Lösung) für die Tuchreinigung gaben viele Hersteller als Option an, um bei Vorhandensein von Zement den Pressbetrieb aufrecht erhalten zu können.

\section{Großmaßstäbliche Versuche zur Entwäs- serung}

Auf Basis der Voruntersuchungen und dem Ergebnis einer ersten Kostenabschätzung [7] wurden Dekanter und Kammerfilterpressen als zielführende Apparate für die Druckentwässerung identifiziert und Firmen vom Projektteam für eine Pilotversuchskampagne bei ausgesuchten Herstellern ausgewählt. Um die Vergleichbarkeit der Daten zu gewährleisten, wurde sichergestellt, dass alle Apparatehersteller das entwässerungstechnisch aufwändigste Material (tonigschluffige Probe) und Zement in derselben Qualität und in ausreichender Menge zur Verfügung gestellt bekamen.

Im Versuchsplan wurden die zu testenden Rezepturen bezüglich Feststoffanteil $(300 \mathrm{~g} / \mathrm{l}, 450 \mathrm{~g} / \mathrm{l}$ und $600 \mathrm{~g} / \mathrm{l})$ und Mengenanteil an Zement an der Trockensubstanz (0, $12,5 \mathrm{~m} \%, 25 \mathrm{~m} \%$ ) vorgegeben. Den Mitarbeitern des Lehrstuhls für Aufbereitung und Veredlung kam die Aufgabe zu, die Versuche zu begleiten, an der Umsetzung der Rezepturen mitzuarbeiten, die Beprobung separat vorzunehmen, die Versuche und die "Gleichheit" der Aufgabeproben zu dokumentieren und allfällig aus der Diskussion mit den Herstellern resultierende Ergänzungen bzw. Abänderungen des Versuchsplans zu beurteilen und vorzunehmen.

Tab. 2 zeigt den Vergleich im spezifischen Filtrationswiderstand und den Kennwerten der Kornverteilung der bei den Versuchen verwendeten Proben.

Tab. 3 zeigt im Vergleich die Maschinendaten und Versuchseinstellungen während der Pilotversuche bei den beiden Anbietern von Dekantern. Der Versuchsaufbau rund um die Dekanter war sehr ähnlich, bestehend aus einer Flockungsmittelstation zur exakten Dosierung einer Flockungsmittellösung in den Aufgabetrübestrom über Exzenterschneckenpumpen, einem Trübevorratsgefäß von etwa $1 \mathrm{~m}^{3}$ Fassungsvermögen mit einem Rührwerk zur Vorbereitung und Homogenisierung der Aufgabetrübe und dem eigentlichen Dekanter (Abb. 5).

Aus den insgesamt 27 Versuchen nach dem zuvor angeführten Versuchsschema wurden folgende Erkenntnisse gewonnen:

- Die effektive Konzentration des Aufgabestromes nach Zumischen des Flockungsmittelstromes überstieg in keinem der Versuche $500 \mathrm{~kg} / \mathrm{m}^{3}$. Die geforderten $600 \mathrm{~kg} / \mathrm{m}^{3}$ waren mit den Dekantern nicht darstellbar, weil es zu Problemen beim Austrag kommt.

- Die geforderten Restfeuchten im Kuchen lagen im Dekanter 1 mit 30\% etwas über jenen von Dekanter 2 mit $35 \%$ bei Verarbeitungszeiten kleiner als eineinhalb Stunden.

- Liegt die Verarbeitungszeit unterhalb von 1,5h, ist der Restfeuchtegehalt unabhängig vom Zementgehalt.

- Bei Erhöhung der Rührdauer zementhältiger Suspension (18h, $6 \%$ Zement) steigt der Restfeuchtegehalt im Kuchen (37\%). Dieser Effekt ist unabhängig vom Entwässe-

\begin{tabular}{|llll}
\hline Kennwert & Dekanter 1 & Dekanter 2 & Kammerfilterpresse \\
\hline Spezifischer Filtrationswiderstand $\left[1 / \mathrm{m}^{2}\right]$ & $3,59 \times 10^{15}$ & $3,93 \times 10^{15}$ & $3,56 \times 10^{15}$ \\
\hline Standardabweichung $\left[1 / \mathrm{m}^{2}\right]$ & $4,88 \times 10^{14}$ & $1,61 \times 10^{14}$ & $5,40 \times 10^{14}$ \\
\hline Korngröße bei: & Malvern Laser Granulometer & 0,55 \\
\hline D-10\% $[\mu \mathrm{m}]$ & 0,38 & 0,53 & 4,91 \\
\hline D-50\% $[\mu \mathrm{m}]$ & 5,23 & 5,01 & 14,25 \\
\hline D-75\% $[\mu \mathrm{m}]$ & 15,9 & 14,56 & 31,52 \\
\hline D-90\% $[\mu \mathrm{m}]$ & 35,57 & 32,86 & \\
\hline
\end{tabular}




\begin{tabular}{|lll|}
\hline $\begin{array}{l}\text { TABELLE } 3 \\
\text { Kenndaten und Versuchsbedingungen der in den Pilotversuchen eingesetzten Dekanter }\end{array}$ & $\begin{array}{l}\text { Dekanter 2 } \\
\text { Dekanter 1 } \\
\text { Typ DP 265-422 }\end{array}$ & Typ Z23-3/441 \\
\hline Trommeldurchmesser $[\mathrm{mm}]$ & 260 & 230 \\
\hline Trommellänge $[\mathrm{mm}]$ & 1155 & 690 \\
\hline Drehzahl Trommel $\left[\mathrm{min}^{-1}\right]$ & 3700 & 3500 \\
\hline Differenzdrehzahl $\left[\mathrm{min}^{-1}\right]$ & 30 & $3,8-12$ \\
\hline Volumenstrom Aufgabe $\left[\mathrm{m}^{3} / \mathrm{h}\right]$ & 1 & $0,4-0,8$ \\
\hline Massekonzentration $\left[\mathrm{kg} / \mathrm{m}^{3}\right]$ & $300-600$ & $300-450$ \\
\hline Flockungsmittel $(\mathrm{FM})$ & Kationisch (PK 65H) & Anionisch (Magnafloc 155) \\
\hline $\begin{array}{l}\text { Gehalt Wirkstoff in der Flockungsmittellö- } \\
\text { sung [\%] }\end{array}$ & 0,20 & 0,20 \\
\hline Volumenstrom FM $\left[\mathrm{m}^{3} / \mathrm{h}\right]$ & $0,12-0,24$ & $0,01-0,016$ (max. 0,1) \\
\hline
\end{tabular}

rungsapparat. In Zeitreihenversuchen mit der Kammerfilterpresse wurde dieser Effekt ebenfalls beobachtet.

- Flockungsmittel verbessern die Zentratqualität bereits in geringer Dosierung $(200 \mathrm{~g} / \mathrm{t})$ und nehmen je nach Art und Dosierung Einfluss auf die Restfeuchte. Die kationischen Flockungsmittel in der hohen Dosierung (440-1670 g/t) bewirkten keinerlei Veränderung der Restfeuchte im Vergleich zum Nullversuch, während das anionische Flockungsmittel in der geringen Dosierung zu einer Erhöhung der Restfeuchte unter sonst gleichen Bedingungen führte.

Die Pilotversuche zur Wirkung der Kammerfilterpresse wurden mit einer manuell zu bedienenden SeitenholmKammerfilterpresse mit 3 Platten einer Fläche von $400 \mathrm{~cm}^{2}$ $(20 \times 20 \mathrm{~cm})$ durchgeführt. Die beiden Kammern mit einem Filtertuch (Typ Tecnoidea Rilsan 11272R), die über einen Hydraulikzylinder unter Druck gehalten wurden, beschickte eine händisch zu bedienende Membrankolbenpumpe mit Trübe. Ein Pressdruck von 6 bar war erzielbar. Als Messwert wurde die Filtratmenge über die Zeit aufgenommen und die Restfeuchte des Kuchens gemessen (Abb. 6).

Bei einer Trübeverarbeitungszeit unterhalb einer halben Stunde wurden unter obigen Bedingungen Restfeuchten zwischen 20 und $28 \%$ erzielt und damit die mit den Dekantern erzielten Ergebnisse übertroffen. Dabei lagen die Feststoffaufgabekonzentrationen in einem für Kammerfilterpressen ungünstigen Bereich $\left(300 \mathrm{~kg} / \mathrm{m}^{3}\right)$. Wie der Abb. 6 zu entnehmen ist, bringt jedoch erst das Vorhandensein von Zement die Filtration in wirtschaftlich relevante
Filtrationszeiten. In Vorversuchen wurde gezeigt, dass zwar Flockungsmittel auch verkürzend auf die Filtrationszeit wirken, aber in deutlich geringerem Ausmaß.

Mit dem Zementanteil steigt allerdings der Restwassergehalt im Filterkuchen unter sonst gleichen Bedingungen. Variiert man die Rührdauer bis zu $48 \mathrm{~h}$, zeigte sich, dass mit zunehmender Rührzeit der Suspension vor dem Verpressen der Restfeuchtegehalt im Filterkuchen steigt, und zwar umso höher, je geringer die Feststoffkonzentration und je höher der Zementgehalt im Feststoff ist (Rührzeit $48 \mathrm{~h}$ und Feststoffkonzentration $300 \mathrm{~kg} / \mathrm{m}^{3}: 44 \%$ Restfeuchte bei $12,5 \%$ Zement, $47 \%$ Restfeuchte bei $25 \%$ Zement im Feststoff). Gleichzeitig geht aber auch die Filtrationszeit stark zurück (bis zu $50 \%$ ).

Flockungsmittel verbesserten die Filtrationszeit und die Restfeuchte. Die Versuche in Pilotpressen sind unter Einbeziehung einer Erhöhung der Filtrationszeit um 2-7 min auf die Filterzykluszeit umlegbar zur Auslegung von Großanlagen.

Als Endergebnis aller Überlegungen und Versuche konnte erkannt werden, dass sowohl Kammerfilterpresse als auch Dekanter die Anforderungen zur Entwässerung des DSV-Rücklaufs mit dem höchsten Entwässerungswiderstand auch bei ungünstigen Feststoffkonzentrationen erfüllen. Zement nimmt bei rascher Verarbeitung im Fall der Dekanter keinen Einfluss, im Fall der Kammerfilterpressen führt er zu einer Verbesserung der Filtrationseigenschaften. Eine Erhärtung von zementhältigen Sedimenten tritt über einem w/z-Wert von 1,5 nicht auf. Ein Ansteifen des DSV-Rücklaufs kann durch Rühren verhindert werden, aller-
Abb. 5: Bilder derVersuchsaufbauten von Dekanter 1 (links) und Dekanter 2 (rechts)
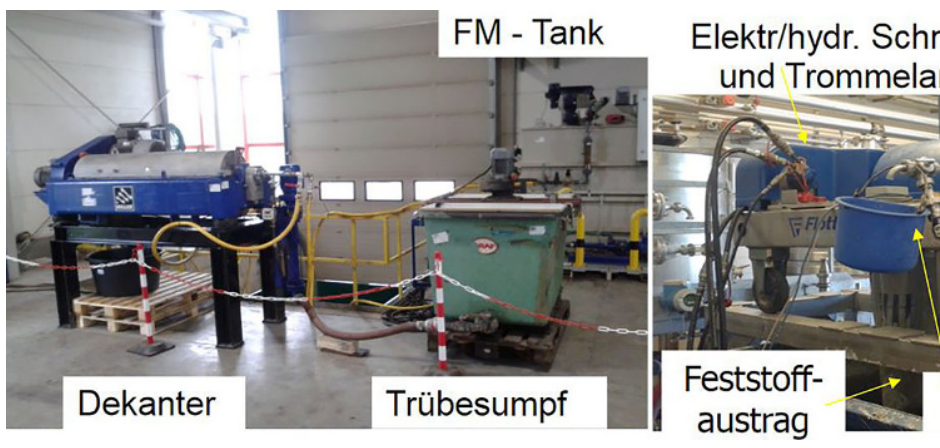

Zentrat
Dekanter

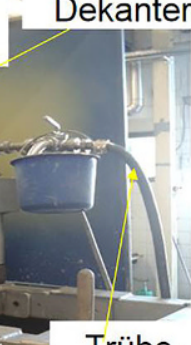

Trübeaufgabe 
Abb. 6: Versuche mit der Kammerfilterpresse. Akkumuliertes Filtratvolumen über dieZeit

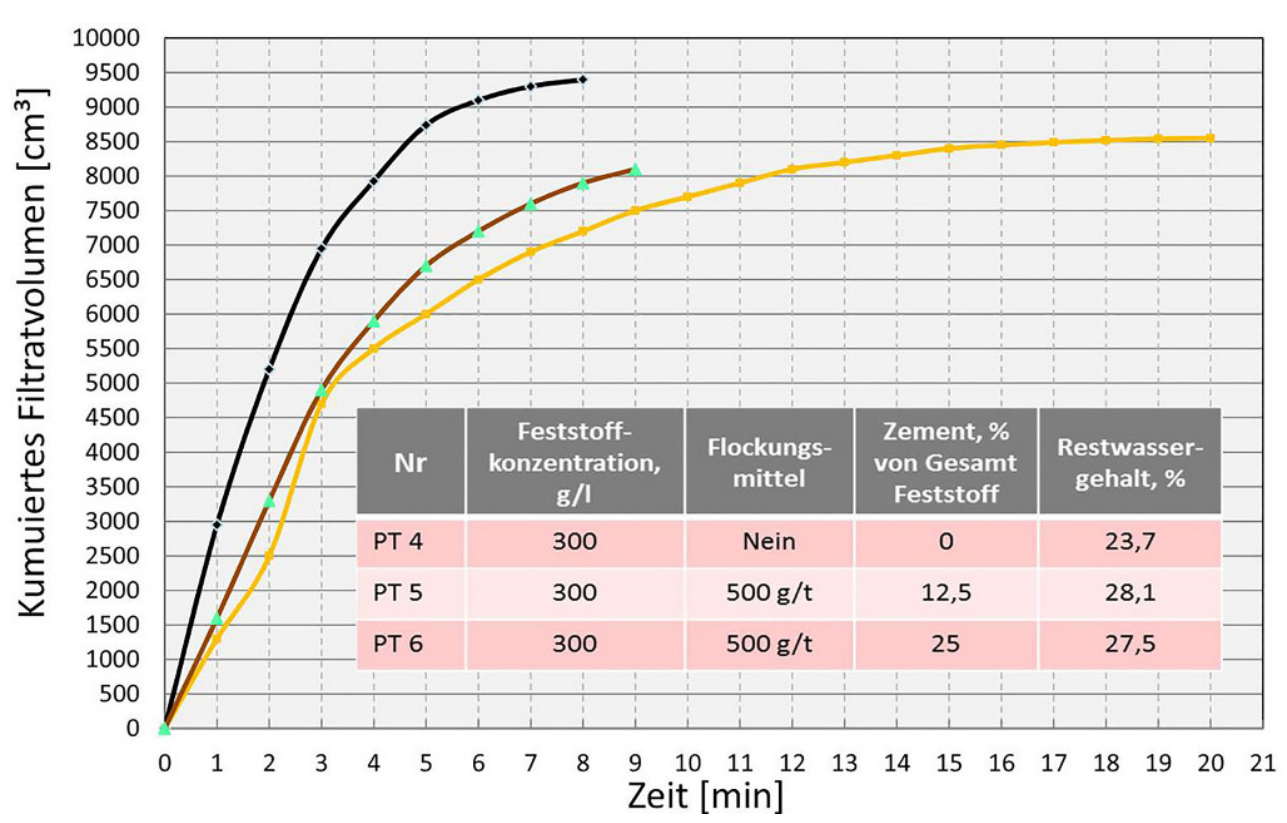

dings bleibt der Rücklauf auch ohne Scherkrafteinwirkung für mindestens $8 \mathrm{~h}$ pumpbar. Damit ist die Möglichkeit gegeben, (ausbaggerbare) Rücklaufbecken einzurichten, die einerseits als Puffer vor der eigentlichen Entwässerung dienen, in denen andererseits aber auch gröbere Körnungen sedimentieren können. Zum Schutz gegen Verschleiß und zur Erhöhung der Betriebssicherheit der Entwässerungsapparate sollten Körnungen größer $1 \mathrm{~mm}$ durch geeignete Klassieraggregate vor der eigentlichen Entwässerung aus dem DSV-Rücklauf entfernt werden. Zwar verbessert die Zugabe von Flockungsmitteln die Qualität des Klarwassers, diese müssen aber aufgrund der unterstützenden Wirkung des Zements nicht als Filtrationshilfsmittel eingesetzt werden. Durch den einsetzenden Hydrationsprozess des Zements bei Rührzeiten jenseits der $8 \mathrm{~h}$ sind höhere Filterkuchen-Restfeuchten zu erwarten.

\section{Ausschreibung und Ausführung}

In der Rohbauausschreibung wurden den Bietern die Erkenntnisse aus dem Versuchsfeld Untersammelsdorf und aus den durchgeführten und in Abschn. 5 und 6 beschriebenen Entwässerungsversuchen als Kalkulationsgrundlage und als Grundlage für die Detailplanung zur Verfügung gestellt [12].

Das DSV-System und die Art und Ausbildung der Entwässerungsanlage wurde den Bietern für die Ausführung jedoch freigestellt.

Auf folgende Punkte hinsichtlich des DSV-Rücklaufs wurden die Bieter hingewiesen:

- Der DSV-Rücklauf ist entsprechend den Erfordernissen des Rohbauauftragnehmers so zu entwässern, dass ein Transport zur durch den AG beigestellten Baurestmas- sendeponie Peratschitzen und ein entsprechender Einbau dort möglich ist;

- Die Entwässerung sollte auch dazu dienen, das einzubauende Deponievolumen zu reduzieren;

- Beim Füllungsgrad der Transportfahrzeuge sind die Konsistenz des Filterkuchens und die maximale Neigung der Baustraßen zu beachten;

- Das System der Entwässerung muss vor Bauausführung im Labormaßstab getestet werden;

- Die Entwässerungsanlage kann stationär, semistationär oder mobil ausgeführt werden;

Neben den dokumentierten Entwässerungsversuchen wurden den Bietern in den Ausschreibungsunterlagen Vorschläge und Überlegungen des Auftraggebers zur Entwässerungsanlage übermittelt. So wurden u.a. folgende Grundeinheiten der Entwässerung vorgeschlagen:

- Fassung des DSV-Rücklaufs am Ort der Entstehung (Bohrlochmund) und Transport zur Entwässerungsanlage;

- mechanische Entwässerung;

- Zwischenlagerung der entwässerten Produkte;

- Kreislaufwasserwirtschaft

- Filtratwasserbehandlung vor Entlassung in den Vorfluter entsprechend den wasserrechtlichen Erfordernissen.

Aufgrund der prognostizierten schwankenden Kornzusammensetzung, der schwankenden Feststoff- und Zementkonzentration und zur Gewährleistung einer hohen Anlagenverfügbarkeit (geringe Ausfallzeiten aufgrund ungeplanter Anlagenstillstände durch Sedimentation in Rohrleitungen bzw. Verstopfung, übermäßigen Verschleiß u.a.) sollte die Anlagenkonzeption zur mechanischen Entwässerung mehrstufig ausgeführt werden. 
Abb. 7: Übersicht über die Spezialtiefbauarbeiten und Entwässerungsanlage im Baulos 60.3

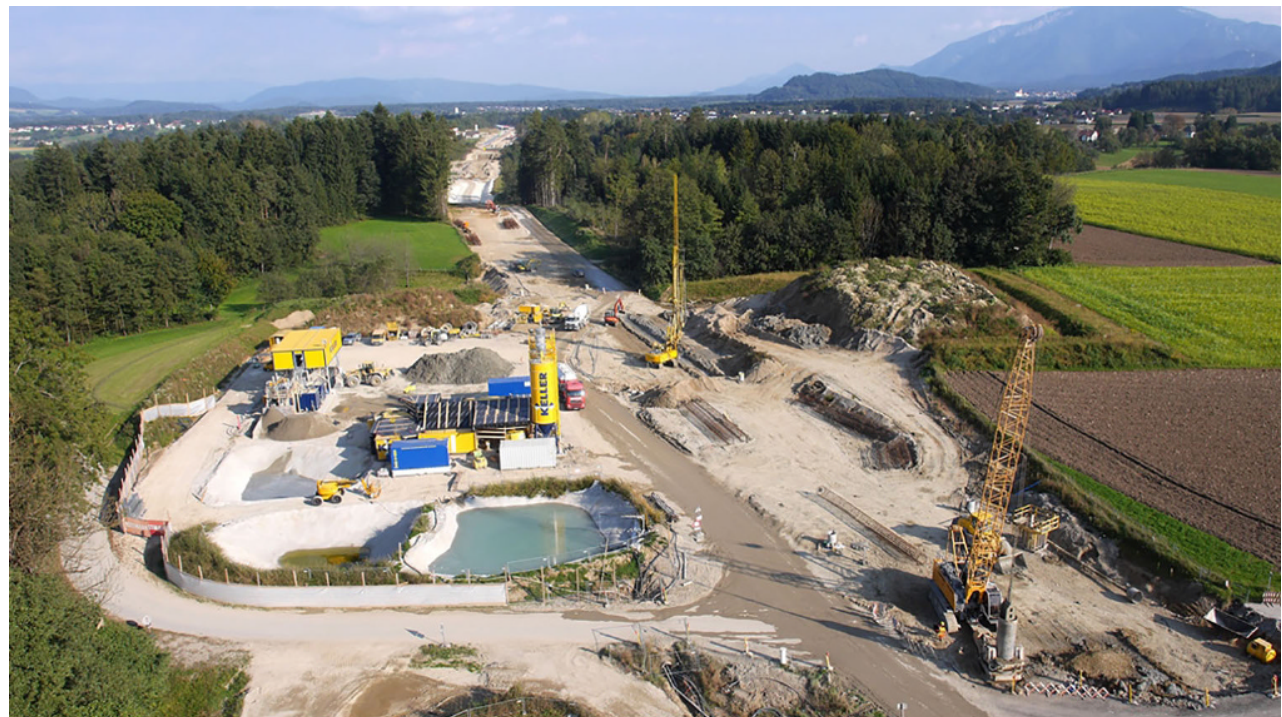

Folgende Prozesskomponenten wurden seitens des Auftraggebers nach Vorgabe des Lehrstuhls für Aufbereitung und Veredlung vorgeschlagen:

- Grobkornabtrennung durch Siebung

- Mittelkorn- zu Feinkornabtrennung durch Stromklassierung im Schwer- oder Fliehkraftfeld (z. B. durch Schraubenklassierer oder Hydrozyklon)

- Feinstkornabtrennung durch mechanische Fest-/FlüssigTrennung der Feinkornsuspension (z. B. durch Dekanter oder Kammerfilterpressen)

Auch die Wahl von Trennschnitten wurde den Bietern freigestellt.

Von den Bietern wurde allerdings verlangt, zusammen mit dem Preisangebot einen technischen Bericht zur Entwässerungsanlage abzugeben, in dem die zum Einsatz kommenden Apparate mit Darstellung der vorgesehenen Verfahrensstufen, des Fließschemas, der Leistungsfähigkeit und Dimensionierung der Einzelkomponenten, der Energieversorgung, des Flächenbedarfes und der Beschreibung der Materialeigenschaften am Prozessende sowie die Puffermöglichkeiten beschrieben wird.

Die Bauausführung erfolgte von 2014 bis 2019 durch eine Arbeitsgemeinschaft der Firmen Kostmann GesmbH und Baresel GmbH. Für die Spezialtiefbaumaßnahmen wurde die Firma Keller Grundbau GmbH als Subunternehmer beauftragt.

In Abhängigkeit der angetroffenen Untergrundverhältnisse und der Menge an Zugabe von Zement als Bindemittel beim DSV-Prozess wurden verschiedene Arten der Entwässerung gewählt.

Bei anstehendem sandigen Seeton konnte eine Entwässerung mittels Schwerkraft in Absetzbecken erzielt werden. Der abgesetzte und angesteifte DSV-Rücklauf wurde mittels konventionellem Bagger nach Erreichung der Stichfestigkeit ausgebaggert und erforderlichenfalls im Baustelleinrichtungsbereich zwischengelagert. Beim tonig-schluffigen Seeton erfolgte die Entwässerung zum größten Teil mittels
Kammerfilterpresse und vorgeschaltetem Hydrozyklon, der bei etwa $1 \mathrm{~mm}$ Korngröße trennte.

Zur Anwendung kam eine Kammerfilterpresse der Fa. Tecnoidea Impianti (41 Kammern, 1300.1300.45 mm) (Abb. 7).

Die gesamte Entwässerungsanlage konnte einen Leistungsstrom von ca. $40 \mathrm{~m}^{3} / \mathrm{h}$ gewährleisten. Der Feuchtegehalt des Filterkuchens betrug 25-30\%.

Der Transport des Filterkuchens und der Einbau in die Deponie verliefen weitgehend problemlos.

Mittlerweile sind die Rohbauarbeiten bei beiden Tunnelbauwerken zum überwiegenden Teil abgeschlossen.

\section{Zusammenfassung}

Die Vorgehensweise des Auftraggebers zur Behandlung eines prozesskritischen technischen Problems (Entwässerung von zementhältigen, feinstkörnigen Suspensionen) durch Einbindung von universitären Fachkräften (Lehrstuhl für Aufbereitung und Veredlung) führte durch systematische Untersuchung der Trübe- und Feststoffeigenschaften und der Kombination des technischen Wissens bezüglich möglicher Umsetzbarkeit auf Baustelle, Deponie und Transport und dem Wissen zur Entwässerungstechnik zu einer praktikablen Lösung, die in ihrer Grundstruktur als mehrstufiger Entwässerungsprozess in der Ausschreibung der Rohbauarbeiten den Bietern zur Verfügung gestellt wurde. Die darauf aufbauende Detailplanung und tatsächliche Ausführung der Entwässerungsarbeiten des Auftragnehmers orientierte sich stark an den Überlegungen, die im Vorfeld angestellt wurden. Letztendlich konnte die Entwässerung des DSV-Rücklaufs erfolgreich durchgeführt und damit der Nachweis erbracht werden, dass die vorab angestellten Grundsatzüberlegungen korrekt waren.

Danksagung. Bedingt durch die lange Projektdauer von ca. 2000 bis 2019 ist die Anzahl der am Projekt beteiligten Personen (und damit der Co-Autoren) umfangreich. Bedanken möchten sich die Autoren bei allen Personen, die im 
Laufe der Projektgeschichte mitgewirkt haben, insbesondere Klaus Berger, Roman Heissenberger, Hanns Wagner, Wolfgang Pistauer, Peter Waibel, Markus Mehlführer, Herbert Gaube und Stefan Leitner, dem Personal der Firmen Kostmann GesmbH, Keller Grundbau GmbH und Baresel $\mathrm{GmbH}$ sowie den damaligen Lehrstuhlmitarbeitern Andreas Franz Moser und Jakob Strzalkowsky.

Funding. Open access funding provided by Montanuniversität Leoben.

Open Access Dieser Artikel wird unter der Creative Commons Namensnennung 4.0 International Lizenz (http://creativecommons.org/licenses/ by/4.0/deed.de) veröffentlicht, welche die Nutzung, Vervielfältigung, Bearbeitung, Verbreitung und Wiedergabe in jeglichem Medium und Format erlaubt, sofern Sie den/die ursprünglichen Autor(en) und die Quelle ordnungsgemäß nennen, einen Link zur Creative Commons Lizenz beifügen und angeben, ob Änderungen vorgenommen wurden.

\section{Literatur}

1. Heissenberger, R.; Benedikt, J.; Mauerhofer, G.: Tunnel Untersammelsdorf - erweiterte Planungsstrategie für Stützmaßnahmen im „Seeton”. Beiträge zum 29. Christian Veder Kolloquium, Stützmaßnahmen in der Geotechnik - Bemessung - Ausführung - Langzeitverhalten. Mitteilungshefte der Gruppe Geotechnik Graz, Heft 51 (2014), S. 233-248

2. Benedikt, J.; Bahr, A.; Wagner, H.; Mehlführer, M.; Berger, K.: Tunnel Untersammelsdorf - Herausforderungen und Sondermaßnahmen beim Vortrieb im Seeton Untersammelsdorf Tunnel - Challenges and Special Measures during Tunneling in Lacustrine Clay. Österreichische Ingenieur- und Architekten-Zeitschrift, 163 (2018), Heft 1-12, S. 1-19

3. Benedikt, J.; Berger, K.; Koinig, J.: Aktueller Planungs- und Ausführungsstand der Tunnelkette St. Kanzian. Geomechanics and Tunnelling, 8 (2015), Heft 6, S. 535-546
4. Benedikt, J.; Wagner, H.; Herzeg, T.: Tunnelkette St. Kanzian Tunnelbau unter sehr unterschiedlichen und auch extrem schwierigen Bedingungen. Geomechanics and Tunnelling, 9 (2016), Heft 5, S. $405-415$

5. ÖNORM EN 12716: Ausführung von Arbeiten im Spezialtiefbau Düsenstrahlverfahren. Ausgabe: 2017-05-01

6. Schachinger, T.; Gaube, H.; Krainer, G.: Results from the Untersammelsdorf test field for the planning of tunnelling work / Erkenntnisse aus dem Versuchsfeld Untersammelsdorf für die Gestaltung der Vortriebsmaßnahmen. Geomechanics and Tunnelling 3 (2010), Heft 2, S. 201-214

7. Böhm, A.: Entwässerungsverhalten von Seeton, Versuchsfeld Untersammelsdorf. Untersuchungsbericht des Lehrstuhls für Aufbereitung und Veredlung 018-17/09, Leoben: Montanuniversität, 2009

8. Moser, F: Entwässerung von Suspensionen aus dem Düsenstrahlverfahren, Masterarbeit, Lehrstuhl für Aufbereitung, Montanuniversität Leoben, 2012, S. 25

9. Latal, C.; Klima, K.: Mineralogisches Gutachten 02/2009. Institut für Angewandte Geowissenschaften, TU Graz, 2009

10. Schubert, H.: Aufbereitung fester mineralischer Rohstoffe, Bd.III Leipzig: VDE Verlag für Grundstoffindustrie, 1984, S. 90

11. Strzalkowsky, J.: Trübevorbereitung und Probekörperherstellung für konsolidierte Scherversuche an der Camillo Sitte Versuchsanstalt, sowie Probenahme, Vorbereitung und Begleitung von Entwässerungsgroßversuchen, Bachelorarbeit, Lehrstuhl für Aufbereitung und Veredlung, Montanuniversität Leoben, 2016

12. ÖBB-Infrastruktur AG: Ausschreibung B13995 Baulos 60.3 - St. Kanzian. 2014

Hinweis des Verlags. Der Verlag bleibt in Hinblick auf geografische Zuordnungen und Gebietsbezeichnungen in veröffentlichten Karten und Institutsadressen neutral. 